\title{
Aero structural optimization for sailplane wing in pre- liminary design
}

\author{
Ahmed El Ibrahim ${ }^{1 *}$, Sohayb Abdul Karim ${ }^{2}$, Ibrahim Gov ${ }^{3}$ \\ 1, 2, 3 Department of Aeronautics and Astronautics, Gaziantep University, Gaziantep, Turkey
}

\section{Keywords \\ Aero-structural optimization Multidisciplinary optimization Sailplane wing design Open aero struct}

Received: 19 October 2017 Accepted: 10 January 2018 Published: 13 February 2018

\begin{abstract}
The purpose of this research is to obtain optimized sailplane wing design, which contributes to better performance in terms of range and endurance. This paper presents aerostructural design optimization using Multidisciplinary Design Optimization approach (MDO). This is carried out using Open Aero Struct (OAS), an open-source low-fidelity aerostructural analysis and optimization tool written in Python and developed in NASA's Open MDAO framework. The aerostructural model in OAS coupled a Vortex Lattice Method for aerodynamic with a 1-D FiniteElement Analysis for structure, to model the sailplane wing. An aerostructural optimization problem was formulated to minimize the Sink Speed, as the objective function, with varying twelve design variables, and subjected to two constraints. An initial simple wing design is used as baseline for the optimization. The findings of the aerostructural optimization indicated that the optimized wing exhibited improvements in both disciplines, resulting a better cross-country performance for the sailplane in terms of range and endurance. The optimized wing achieved increase of $37 \%$ in endurance and $27 \%$ in range.
\end{abstract}

(C) 2018 The Author(s). Published by TAF Publishing.

\section{INTRODUCTION}

Sailplanes are symbolic of aerodynamic efficiency. Many factors contribute to this efficiency, yet the wing is the key factor here. That is why the wing design is the focus of attention for designers. Sailplanes are usually distinguished by high aspect ratio wings in order to achieve high aerodynamic efficiency. At the same time, high aspect ratio is challenging from structural point of view. Therefore, such these inter-disciplinary considerations, call for special design procedures to integrate the aerodynamic efficiency with robust structure in single design. For this reason, the integration between these two disciplines; structure and aerodynamic should be considered and examined in the early preliminary design stage of the wing. An early integration analysis and optimization lead to smoother transition to the detailed design stage.

\section{SAILPLANE FLIGHT AND PERFORMANCE}

\section{A. Soaring and Gliding Flight}

Gliding is the origin of flight, all the early attempts of flying were powerless and mostly depended on wings that emulate the birds. Up to early 1900s, most of these attempts did not last more than few minutes. However, they were the inspiration for present sailplanes, which are capable of flying for tens of hours and hundreds of miles without one millilitre of fuel. This outstanding performance came as a result of incorporating modern technologies and theories in sailplane design. Soaring is heavier-than-air flight without the use of thrust. As a result, only three forces act on the sailplane; Lift, Drag and Weight (Figure 1). Although a sailplane is not powered, there is still a need to obtain thrust. It does this by converting the potential energy into kinetic as it glides downward, trading height for distance.

\footnotetext{
*Corresponding author: Ahmed El Ibrahim

†email: alibrahim.ahmad88@gmail.com
} 


\section{B. Sailplane Flight Performance}

The performance of sailplane can be evaluated by its maximum range or maximum endurance. However, each one of these two figures is related to performance parameter. The maximum range corresponds to maximum glide ratio. At the same time, maximum endurance corresponds to minimum sink speed. In order to understand these parameters better, we assume a sailplane at equilibrium $(L=W)$, yields:

$$
\begin{aligned}
& D=W \sin (\gamma) \\
& L=W \cos (\gamma)
\end{aligned}
$$

Where $D$ is the drag force, $W$ is the sailplane weight, $L$ is the lift force, and $\gamma$ is the flight path angle, which in a steady state glide called the glide angle. Dividing Equation 1 on 2:

$$
\tan (\gamma)=D / L=C D / C L
$$

This angle is independent of the weight and its lowest value corresponds to the higher $L / D$ ratio:

$$
L / D=C L / C D
$$

The lift-to-drag ratio is known as the glide ratio. This is the most important parameter in sailplane performance. Most sailplanes have maximum glide ratios between 20 and 50 and The corresponding glide angles lie between $\sim 3$ and 1 $\operatorname{deg}[1]$.

1) Gliding range: Assuming the sailplane descents from initial height $\left(\mathrm{h}_{1}\right)$ to another height $\left(\mathrm{h}_{2}\right)$, we can define the glide range, $R$, as the horizontal distance a sailplane covers between these two altitudes. It is calculated as following:

$$
R=\frac{h_{1}-h_{2}}{\tan (\gamma)}=\frac{C L}{C D}\left(h_{1}-h_{2}\right)
$$

This equation states clearly that maximum range $\left(R_{\max }\right)$ is corresponded to maximum glide ratio $(C L / C D)_{\max }$.

2) Gliding endurance: The glide endurance, $T$, is the time a sailplane takes to descent between two given altitudes and it is calculated as following:

$$
T=\frac{\Delta h}{v_{s}}
$$

The maximum endurance in still air is obtained by maintaining the sink speed minimum $[2,3,4]$.

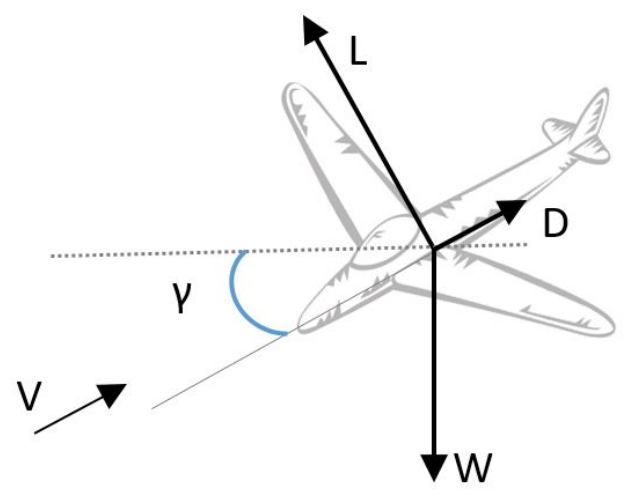

Fig. 1. Forces on a sailplane

With the assumption that the glide angle is small, the sink speed can be written as following:

$$
V_{s}=-V \frac{D}{W} \approx-V \frac{D}{L}=\sqrt{\frac{W}{\frac{1}{2} \rho s}} \frac{C D}{\frac{3}{C_{L}^{2}}}
$$

Where $\mathrm{S}$ is the reference area of the wing and $\rho$ is air density. This equation, which relates airspeed with wing loading $(W / S)$ and drag and lift coefficients, is of fundamental importance to sailplane performance analysis. To have minimum sink speed, both of the two quantities wing loading $(W / S)$ and $C D / C L^{3 / 2}$ should be minimum.

\section{MULTIDISCIPLINARY DESIGN OPTIMIZATION}

\section{A. MDO Definition and Application}

MDO is a field of engineering that focuses on the use of numerical optimization techniques for the design of engineering systems, which involve multiple disciplines or subsystems (e.g., Aerodynamics, Structure, Control, ...etc.). The need for MDO came from the fact that the performance of a multidisciplinary system is driven not only by the performance of the individual disciplines but also by their interactions. In the process of design optimization, a well-defined optimization problem is one of the important roles of the designer besides other important numerical choices.

\section{B. MDO Problem Formulation}

MD0 problem formulation is the selection of design variables, constraints, and objectives. Normally, this is the difficult part of the optimization.

1) Objective function: The objective function, $f$, is the numerical value that is to be maximized or minimized. The choice of objective function is governed by the nature of problem. Many methods work only with single objective. For the case of this research, the objective function is 
aerostructural objective, the sink speed. Minimization of sink speed, means minimization of both drag coefficient and structural weight of a sailplane.

2) Design variables: The design variables, $\mathrm{x}$, are the parameters that are varied in specific range by the optimizer in order to maximize/minimize the objective function. Design variables are often bounded by upper and lower values. Many types of design variables could be used, such as quantitative and qualitative, local and global, continuous and discrete.. etc.

3) Constraints: The constraints are conditions to be satisfied to keep our design logical and feasible. There are two main types of constraints, equality constraint; the condition is restricted to equal a fixed quantity. The other type is, inequality constraint; the condition is required to be greater/smaller or equal to a certain quantity.

4) Optimization problem statement: Mathematically, the Optimization problem is usually given as:

Minimize $f(x)$ by varying $\mathrm{x}$; from $x_{1}$ to $x_{2}$

Subject to $C_{1}=0 \& C_{2} \geq 0$

$f$ : Objective function, (e.g., structural weight or drag).

$x$ : Vector of design variables, (e.g., twist, thickness ..); $x_{1}$ is the lower bound and $x_{2}$ is the upper bounds.

$C_{1}$ : Vector of equality constraints (e.g., lift = weight).

$C_{2}$ : Vector of inequality constraints (e.g., structural failure).

\section{MDO FRAMEWORKS}

Many MDO frameworks and architectures have been developed and evaluated during few past decades. Set of requirements have been put forward by [5] for MDO framework to possess, including; Architectural Design, Problem Formulation, Problem Execution and Information Access. A selection of architectures is subjected to number of aspects, like for instance, the number of the disciplines involved, the number and type of design variables, the level of fidelity and the optimization methods and algorithms. The MDO architectures can be classified in: single-level methods and multilevel methods. Single-level methods, like Individual Discipline Feasible (IDF) or Multi-Disciplinary Feasible design (MDF), include only one optimizer at a system-level, which runs a system analysis in each step and has authority over the global system [6]. In this paper, OAS is used, which is a tool developed within OpenMDAO framework. Below, an introduction of these two framework is presented.

\section{A. Open MDAO Framework}

Open MDAO [7] MDAO stands for Multidisciplinary Design Analysis and Optimization is an open-source highperformance computing platform for systems analysis and multidisciplinary optimization, developed by NASA and written in Python. The main motivation for developing this framework, was to aid in the design of unconventional aircraft. However, the general structure and methods can be applied to solve any number of engineering-related design problems [7].

OpenMDAO provides a library of sparse solvers and optimizers, and works with both gradient-free (e.g., genetic algorithm, particle swarm) and gradient-based optimization methods. One other hand, OpenMDAO has ability to subdivide a problem into groups or components and link their inputs and outputs, made it easier to decompose large and complex problems. OpenMDAO has been used to solve MDO problems in satellite design [8], wind turbine design [9], aircraft design [10] and aircraft trajectory optimization [11].

\section{B. OAS Framework}

OAS an open-source coupled aerostructural analysis and design optimization tool [12]. OAS developed within OpenMDAO framework. The Aerostructural model within OAS couples aerodynamic solver, which uses the Vortex-Lattice Method (VLM) with structural solver, which uses FfiniteElement Analysis (FEA) using six Degree-Of-freedom (DOF) spatial beam elements. OAS code serves as educational and research MDO tool. It has been used in graduate-level MDO courses at ISAE-SUPAERO (the University of Toulouse's Institute for Aerospace Engineering) and the University of Michigan [12]. As well as, It has been used in research fields as a realistic testbed application by $[13,14,15]$.

The default aerostructural optimization problem in OAS is the fuel-burn minimization using the Breguet Range Equation. Yet, definition of other objectives and constraints, is still applicable due to the modularity nature of the code. As in our case, a function for sink speed was added as objective function. The sink speed Equation 7, is function to both structural variables i.e., weight and aerodynamic coefficients i.e., lift and drag coefficients. Thus, the minimum sink speed corresponds with minimum weight and minimum drag at fixed lift.

\section{AEROSTRUCTURAL WING MODEL}

\section{A. Aerodynamic Model}

The aerodynamic model in OAS, calculates the total lift and drag. However, the induced drag is calculated using Vortex Lattice Method (VLM), whereas, the viscous drag is calculated using flat-plate-based estimation. In VLM, the wing is segmented into a system of panels, each panel is represented by a horseshoe vortex (Figure 2). Each horseshoe vortex has strength and consists of bound vortex, which 
placed at the quarter panel chord, and two trailing vortices. The horseshoe vortex induces a velocity at a control point that placed at $3 / 4$ of the panel chord at its mid-section. This velocity is calculated using the Biot-Savart law [16]. Then, summing all control points together on the wing in order to produce a set of linear algebraic equations for the horseshoe vortex strengths. Taken into account, that the boundary condition of no flow through the wing is satisfied i.e., velocity normal to the panel must be zero.

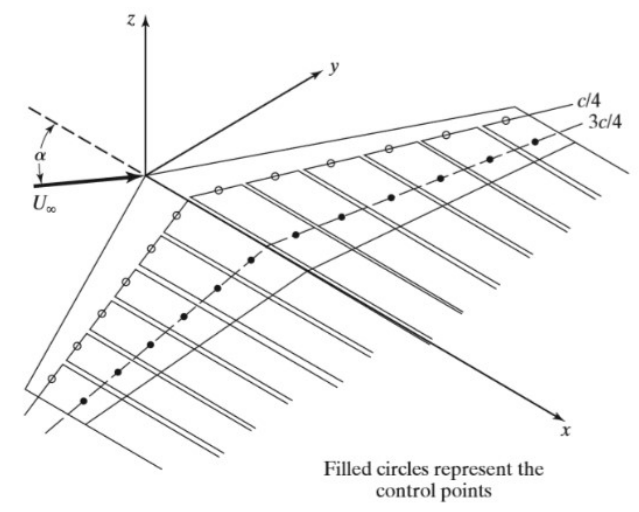

Fig. 2. Distributed horseshoe vortices over a wing [16]

The vortex strengths are related to the wing circulation and the pressure differential between the upper and lower wing surfaces. Integrating the pressure differentials results the total forces and moments.

\section{B. Structural Model}

OAS simulates the wing structurally as a spatial beam element. A tubular beam is used to represent the structure side of the sailplane wing. Mathematically, a FEM pproach that uses spatial beam elements is employed, resulting in six DOF per node. As Figure 3 illustrates, each element has 12 DOFs in total, including both translational and rotational displacements, which are aligned with the local coordinate frame.
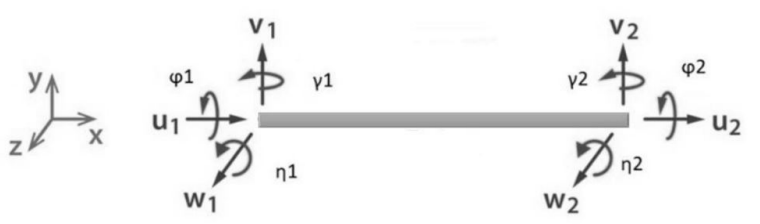

Fig. 3. 6-DOF Spatial beam element

After setting up the global stiffness matrix $K$ (12X12), OAS solves the linear system $K u=f$, where $u$ is the displacements and rotations vector at the nodes (12X1), and $f$ are the forces and moments acting at the nodes.

Further elaboration on both aerodynamic and structural theories in OAS, is provided by [12].

\section{Aero-Structural Coupling}

To have an aerostructural model, a coupling mechanism between aerodynamic and structural models is needed. In addition to load and displacement transfer scheme.

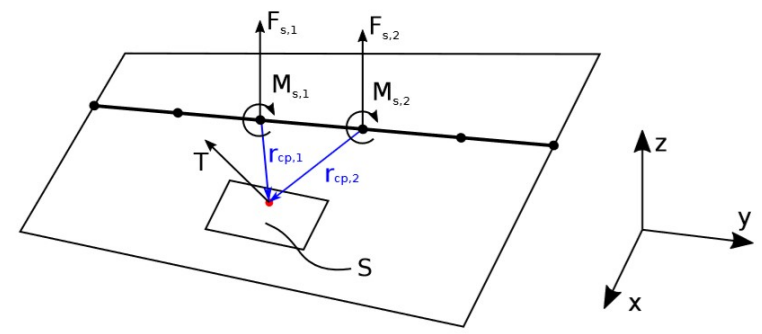

Fig. 4. Load transfer from aerodynamic mesh to structural nodes [12]

1) Load and displacement transfer scheme: The load and displacement transfer process should grantee the accurate translation of the nodal displacement of the structure model to the aerodynamic mesh point displacements. As well as, transferring the loads on the aerodynamic panel to the structural nodes. In OAS, the load and displacement transfer is simplified as aerodynamic and structural models have the same spanwise discretization. Additionally, the transfer scheme satisfies the requirements of being consistent and conservative [17]. Consistency requirement, is that the resultant nodal forces and moments are the same of forces and moments resulted from the pressure distribution for each element. Conservativeness requirement, defined as the virtual work resulted by the load vector over virtual displacements of the structural model, equals the work performed by pressure filed on the aerodynamic mesh.

Demonstration that these two requirements are satisfied in this transfer scheme, is performed by [12].

2) Aerostructural optimization mechanism: The aerostructural model is a combination of the aerodynamic and structural models described above. These two models are structured within the aerostructural system as shown in Figure 5. The aerodynamic model receives the mesh and produce loads, whereas the structural model receives the aerodynamic loads and outputs displacements. This continues until the analysis converges. 


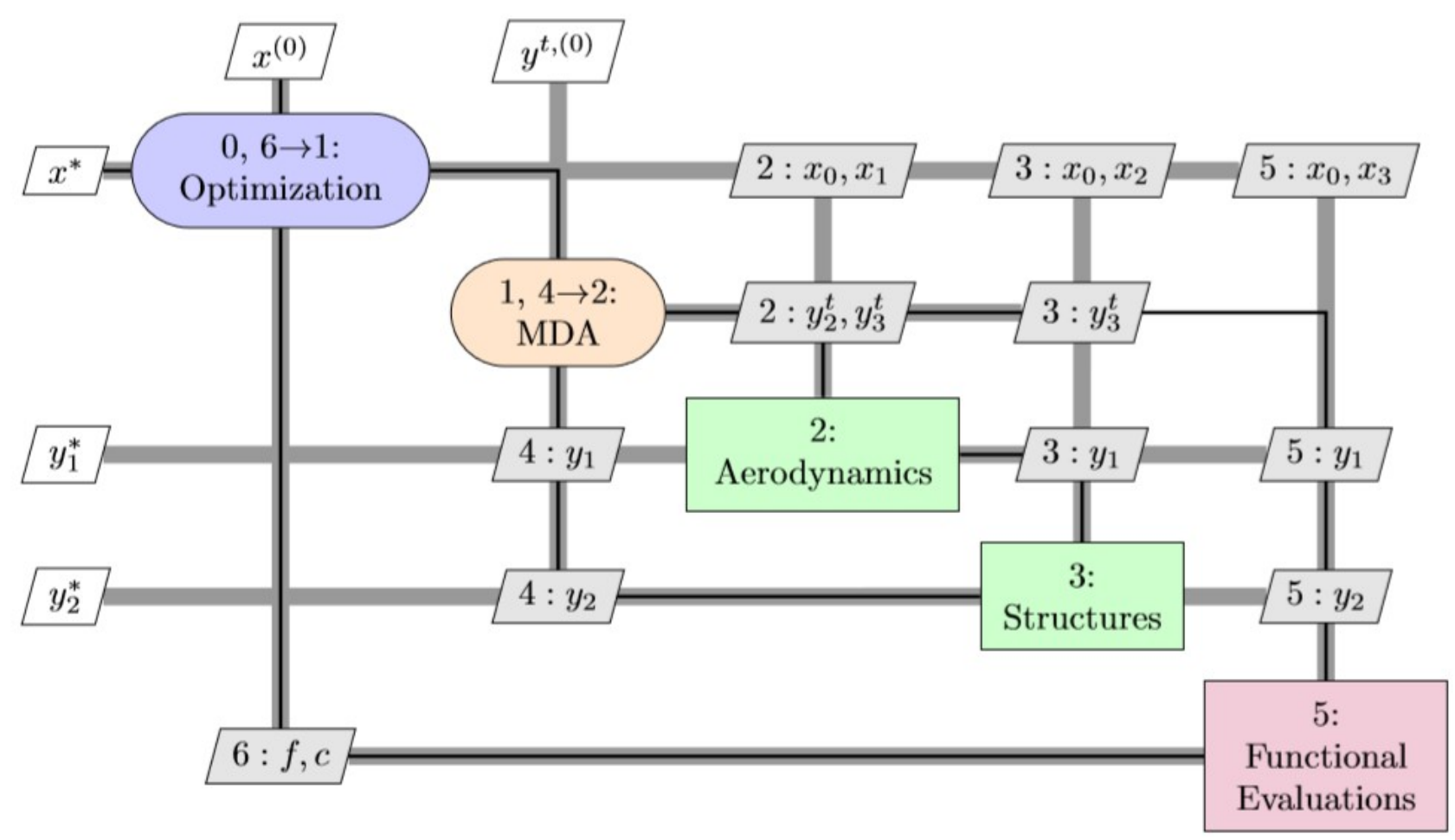

Fig. 5. XDSM diagram for default aerostructural optimization [12]

The Multidisciplinary Analysis (MDA) uses Gauss-Seidel fixed-point iterations to converge the aerostructural system [12]. Figure 5 shows the XDSM diagram [18] corresponding to aerostructural optimization. Here, the input and output vectors, $x$ and $y$, respectively, vary depending on the design variables, objectives, and constraints selected. The $\mathrm{x}$ vectors are design variables and the $y$ vectors are states, where * represents the values at the design optimum.

3) The optimizer: Currently most MDO studies use gradient-based methods [19] as the optimizer used in this paper. The optimizer, used here, is gradient-based algorithm called SLSQP. SLSQP is a sequential least squares programming algorithm [20] that evolved from the least squares solver of Lawson and Hanson. It uses a QuasiNewton Hessian approximation and an L1-test function in the line search algorithm. SLSQP uses Sequential Least SQuares Programming to minimize the objective function of variables and their bounds, and equality and inequality constraints.

\section{METHODOLOGY}

The objective of this paper is to obtain an efficient wing design for sailplane, which contributes to high performance in terms of range and endurance. Thus, this requires maxi- mum glide ratio (CL/CD) for the range, as well as minimum (CD/CL1.5) and minimum weight for sink speed, as mentioned in the performance. Therefore, we have objectives from two disciplines: glide ratio in aerodynamic and weight - precisely, the structural wing weight - in structure. Given that these two disciplines are deeply intersected, the multidisciplinary design optimization approach is selected to do the aerostructural analysis and optimization. The objective function of the aerostructural optimization is the sink speed, Equation 7. Minimization of sink speed, corresponds to minimize both weight and drag. To accomplish this, an objective function component for sink speed was added to OAS code. At the end, the aerostructural optimization for the sailplane wing is as following:

$$
\text { Minimize } \sqrt{\frac{2 W}{\rho s}} \frac{C_{D}}{C_{L}^{1.5}}
$$

w.r.t. Twist, angle of attack, Taper, Beam Thickness subject to $L=W$, Stress failure

Where; $W$ is the sailplane weight, $C D$ is drag coefficient, $C L$ is lift coefficient, $S$ is wing reference area, $\rho$ is air density and $L$ is the lift force. For the case study, an initial wing design is selected as shown Table 1. This wing represents the baseline design for the aerostructural optimization. 
TABLE 1

INITIAL SAILPLANE WING SPECIFICATIONS

\begin{tabular}{lll}
\hline \hline Specification & Value/Description & Unit \\
\hline Planform & Rectangular & - \\
Root chord & 0.7 & $\mathrm{~m}$ \\
Span & 15 & $\mathrm{~m}$ \\
Reference Area & 10.5 & $\mathrm{~m} 2$ \\
Aspect ratio & 21.4 & - \\
Twist & 0 & degree \\
Taper Ratio & 1 & - \\
Airfoil NACA & 633618 & - \\
\hline \hline
\end{tabular}

This wing has simple geometry, in order to capture any changes will tack place after the optimization. However, the span is fixed on 15 (as in standard and 15-meter sailplane class) and the initial aspect ratio is 21.4 .

\section{RESULTS AND DISCUSSION}

\section{A. Setting Up the Aerostructural Problem}

As Equation 8 indicates, to minimize the aerostructural objective function i.e., sink speed, a set of design variables were selected in association with two main constraints. These design variables are:

- Taper Ratio: it varies within the range 0.3 to 1 .

- Twist Angle: five control points over the wing span were set to examine spanwise twist distribution, the angle at each control point ranges from -10 to 15 degree.

- Beam Thickness: five controls points were set in spanwise direction. The thickness is bounded in the range 0.001 to $0.1 \mathrm{~m}$.

- Angle of attack: it varies within the range -10 to 10 degree.

To point out, the aerostructural optimization problem has twelve design variables in total. In addition to two constraints:
- Lift equals weight: means that the wing should produce lift amount that can carry the sailplane weight. Also, it is worth to mention that this constraint used to derive the objective function relation in the first place.

- Failure constraint, is the aggregated beam failure using a Kreisselmeier-Steinhauser (KS) function [21, 22, 23]. For the aerodynamic model, the flight conditions of a crosscountry flight are used. On the other hand, for the structural model, the mechanical properties were based on Aluminium 7075, Table 2.

Now, given that all the conditions for aerostructural problem have been set, an aerostructural optimization for the case study wing is ready to run using OAS code.

By running the aerostructural optimization problem in OAS, the solution converged after 59 iterations and Table 3 summaries the optimization results.

The results of the aerostructural optimization show that the objective is achieved, with the sink speed was minimized. This minimization is resultant of maximization of the glide ratio, as well as, minimization of the structural weight. Equally important, the two constraints also have been fulfilled during the optimization.

TABLE 2

AERODYNAMIC AND STRUCTURAL MODELS SPECIFICATIONS

\begin{tabular}{llll}
\hline \hline Model & Parameter & Value & Unit \\
\hline Aerodynamic & Speed & 25 & $\mathrm{~m} / \mathrm{s}$ \\
& Height & 1,000 & $\mathrm{~m}$ \\
& Angle of attack & 3 & $\mathrm{degree}$ \\
& Air Density & 1.112 & $\mathrm{Kg} / \mathrm{m}^{3}$ \\
Structure & Young modulus & 71.7 & $\mathrm{GPa}$ \\
& Shear Modulus & 26.9 & $\mathrm{GPa}$ \\
& Material Density & 2810 & $\mathrm{Kg} / \mathrm{m}^{3}$ \\
\hline \hline
\end{tabular}




\section{B. Aerodynamic Optimization Findings}

For the aerodynamic aspect of the optimization, the optimizer worked on minimizing CD through ensuring an elliptical lift distribution over the wing, where the elliptical distribution is precisely the optimum distribution for minimum induced drag [1]. For the purpose of that, the optimizer have changed the taper ratio from 1 to 0.3 and associated this with varying the twist angle distribution along the span. As a matter of fact, changing the geometry from rectangular to tapered is quite interesting finding, where in reality, tapered wing is the planform found most frequently on gliders [23, 24].

On the other hand, even though tapering a wing planform gives a number of significant aerodynamic and structural advantages, it also could be challenging regarding wing tip stall unless an appropriate twist distribution is considered. For that reason, after settling on the taper ratio, the optimizer kept changing the twist distribution over the span in a pattern that achieving an elliptical lift distribution, and thereby, minimizing the induced drag. Moreover, the variation of twist was associated with changing the angle of attack as well. Where, the optimizer increased the angle of attack about 4 degrees to meet the required amount of lift. Another interesting finding, the optimizer used the twist angle, and benefited from the cosine-spaced mesh, to bend up the last panel at the tip, forming a winglet-like shape (Figure 7). At the early preliminary design, this could be a good indication to consider at following design stages of the wing.

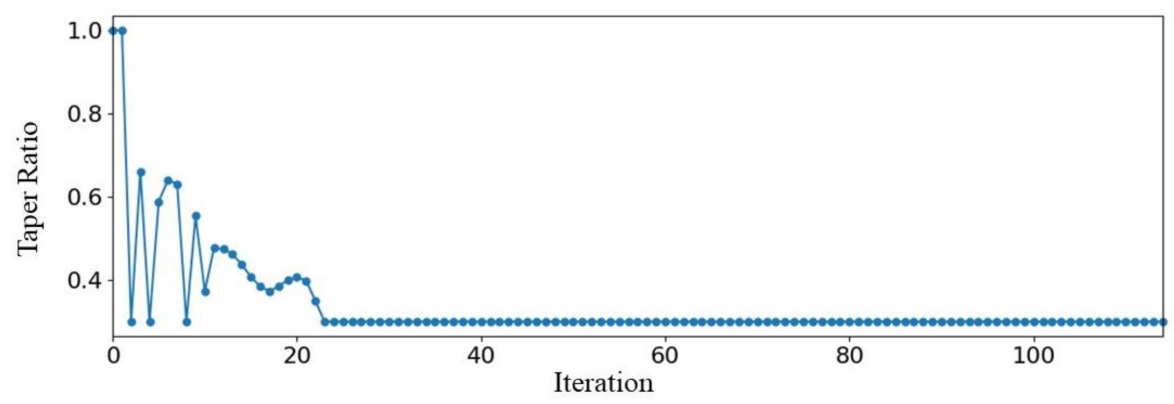

a. Taper ratio during the optimization process.

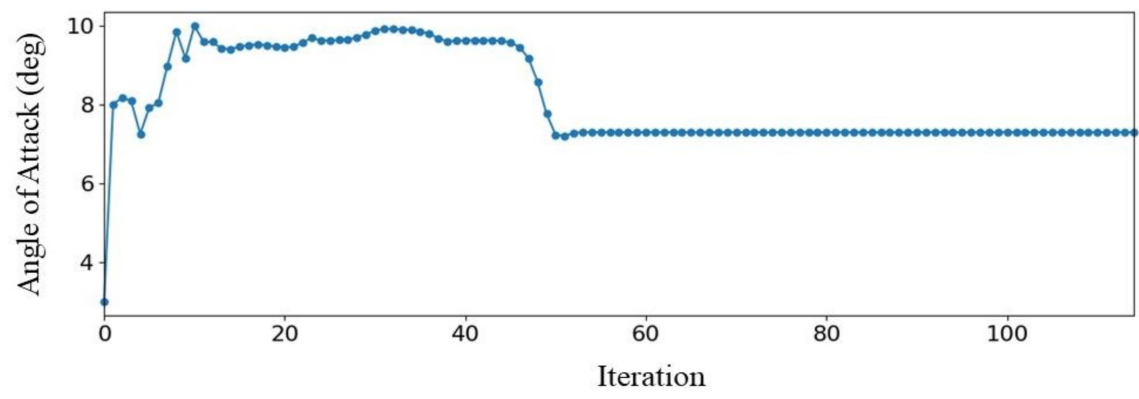

b. Angle of attack during the optimization process.

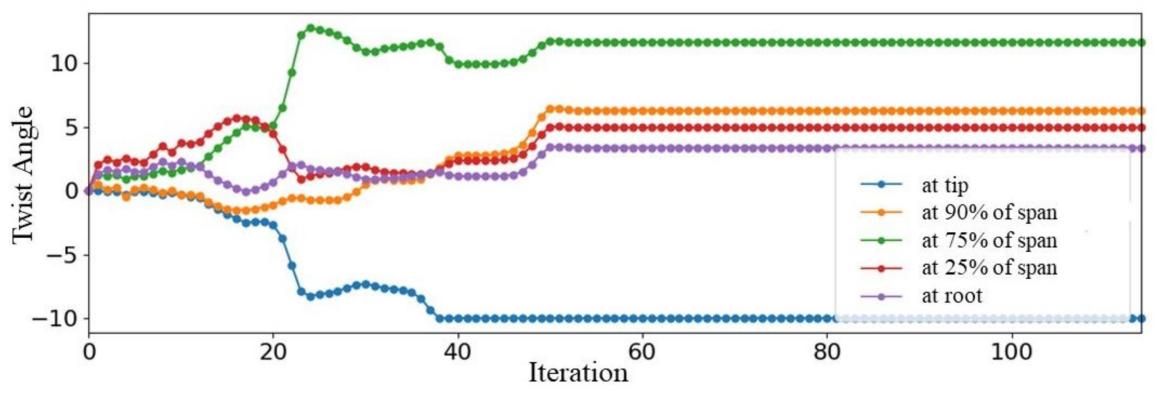

c. Twist angles for the five control points along the span during the optimization process.

Fig. 6. Aerodynamic design variables during the optimization process 
However, the benefits that a winglet provides in performance and handling qualities are being repeatedly proved $[25,26]$. In total, the optimizer has managed to increase $\mathrm{CL} / \mathrm{CD}$ ratio about $27 \%$ more than the initial value of the baseline wing with fulfilling the lift-equals-weight constraint.

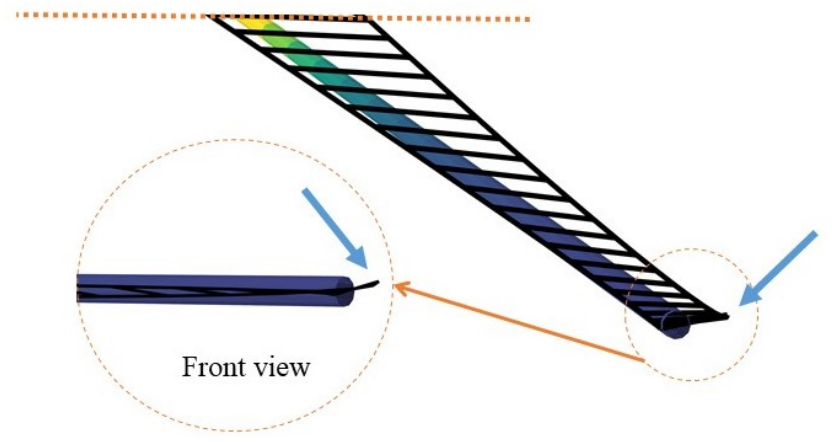

Fig. 7. Bent-up tip of the optimized wing

\section{Structural Optimization Findings}

For the structural aspect of optimization, the objective of this optimization is clearly the weight minimization. Given that the simplicity of structural model, the main factor in weight minimization is reducing thickness over span with fulfilling the failure constraint.

The initial thickness of the structure model is $0.0044 \mathrm{~m}$, which is constant over the span. This thickness distribution gives an initial wing weight of $49 \mathrm{~kg}$. As the structural weight is affected by the lift distribution, the thickness was varying throughout the optimization unevenly from the root to the tip. The largest thickness value appeared at the root as expected, where the bending moment is the highest. Then, it was gradually decreasing from root to tip as Figure 10 illustrates. As a result, the wing structural weight has been reduced more than $50 \%$ with fulfilling the failure constraint.

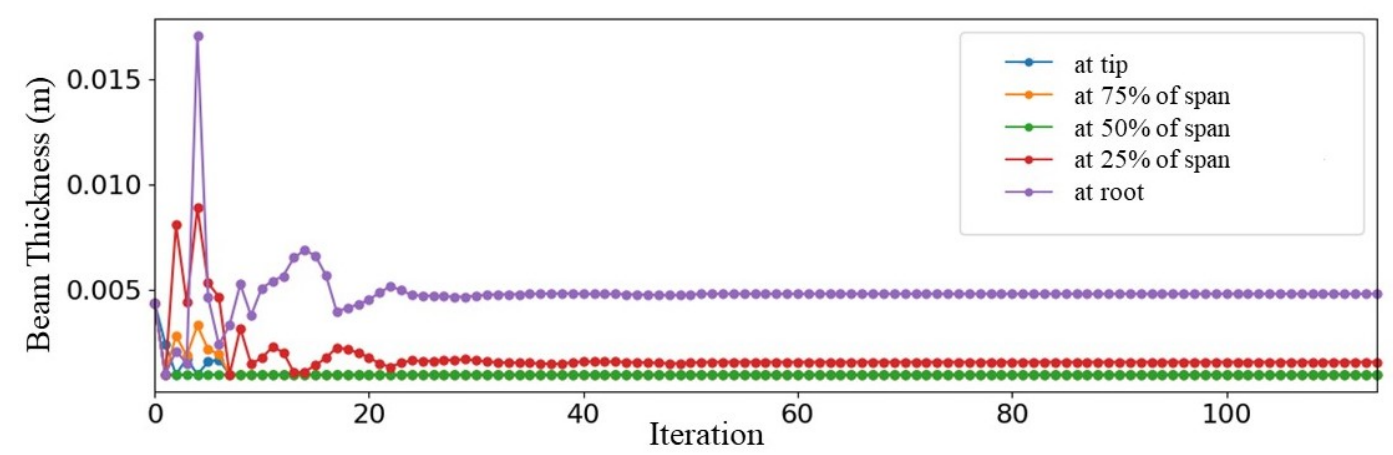

Fig. 8. Beam thickness for the five control points along the span

\section{Aero-Structural Optimization Findings}

As the objective of the aero-structural optimization is minimization of sink speed, this implies minimization of two sub-objectives, the drag and the weight. However, the interdependencies among the aerodynamic and structural design variables drive the optimizer to vary the design variables in a consistent way, which leads to minimum objective function and, at the same time, satisfies the constraints.

By observing the optimization process, one can notice that the optimizer reduced the taper ratio, aiming to increase the aspect ratio, where increasing aspect ratio leads to less induced drag. At the same time, reducing taper ratio leads to reduce the wing area moving outboard from the root. Correspondingly, this reduces the lift as moving to tip. Thus, unloading the wing as moving to tip yields a consistent decrease in beam thickness i.e., less structural weight. Similarly, for twist, the optimizer changed the twist distribution in a consistent way, aiming to have elliptical lift distribu- tion, which evidently leads to minimum induced drag and less structural weight. This is more obvious at the wing tip, where the tip is twisted down (washout). Wings often have less incidence at the tip than the root (washout) to reduce structural weight and improve stalling characteristics [24]. As (Figure 6c) shows, the optimizer twisted down the tip by 10 degrees, leading to decrease the local angle of attack at tip, which means less local lift at the tip. As a consequence, lesser beam thickness at the tip would be enough to provide adequate stiffness. This, in turn, leads to reduce the structural weight.

Using range equation, Equation 5 and endurance Equation 6, one can capture the improvements of optimized wing on the sailplane performance in cross-country flight, Table 3. For the range, the optimized wing showed an improvement of $27 \%$ - i.e., additional $10.5 \mathrm{~km}$ - better than the baseline wing. Likewise, for the endurance, the optimized wing increased the endurance by $37 \%$ - i.e., additional 14 minutes - better than the baseline wing. 


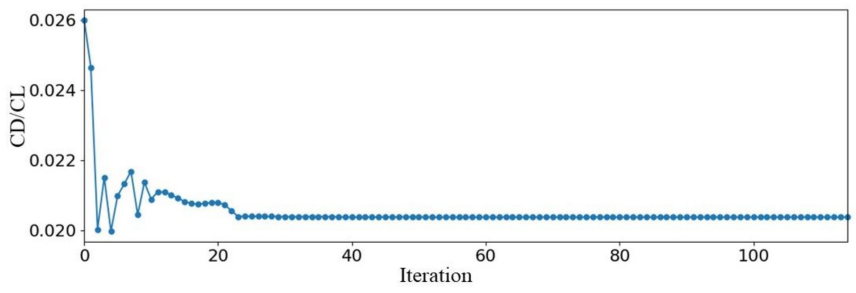

a. Inverted glide ratio variation during the Optimization.

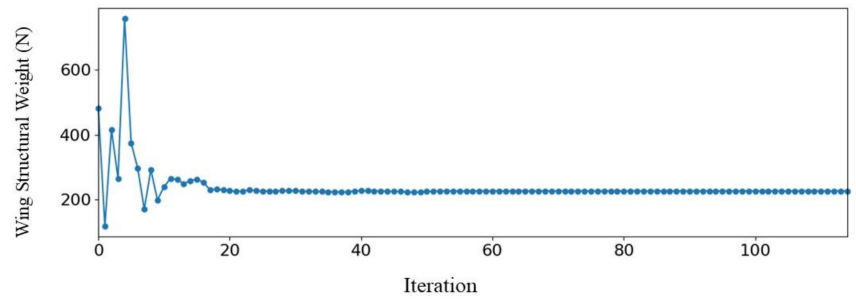

b. Wing structural weight variation during the Optimization.

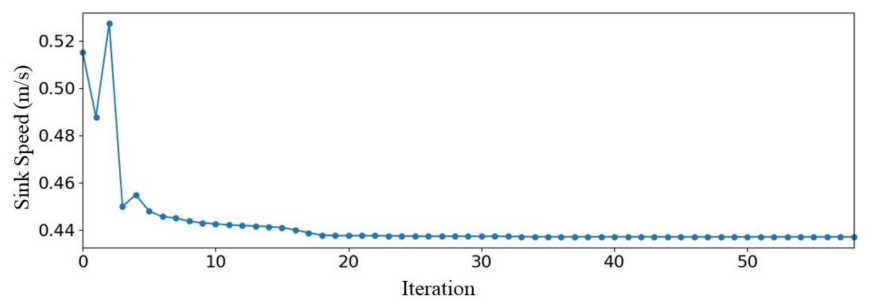

c. Sink Speed (the objective) variation during the Optimization.

Fig. 9. Beam thickness for the five control points along the span

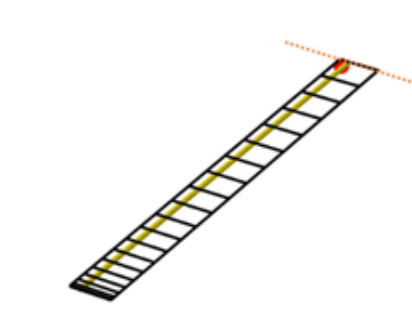

Iterations $=0$
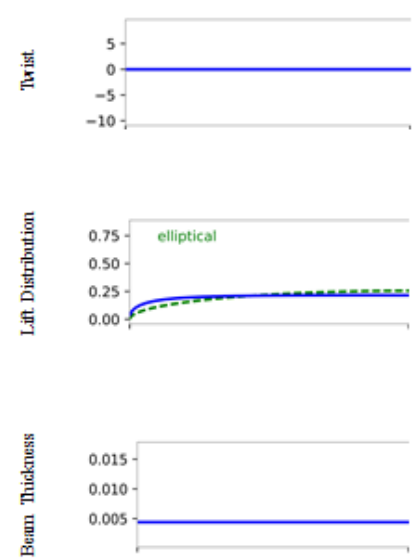

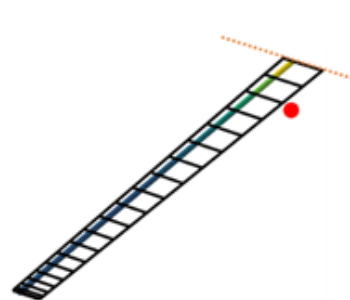

Iterations $=5$
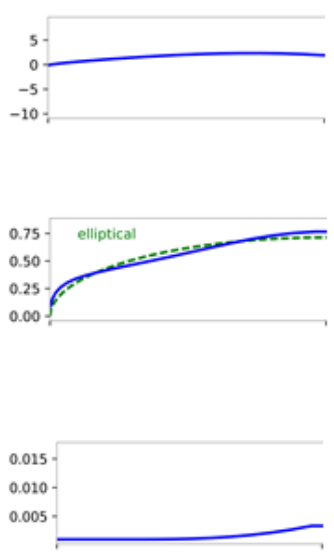

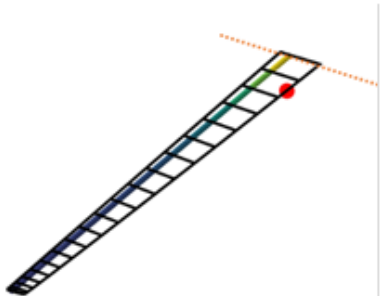

Iterations $=17$

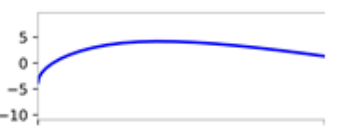

$$
-10
$$
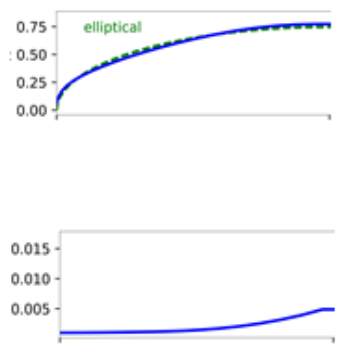

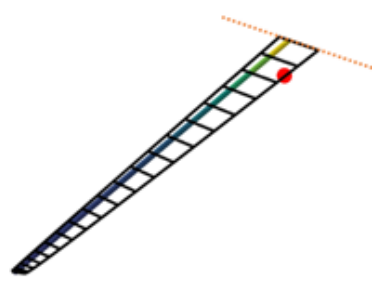

Iterations $=59$

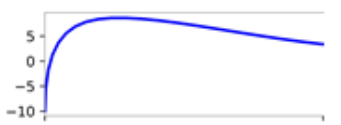

0.75 - elliptical

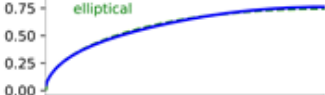

0.015 .

0.015

0.010

0.005 -

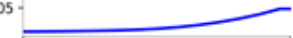

Fig. 10. Aerostructural optimization at four iteration points, starting from the initial design (on the left) and ending at the optimized design (on the right) 
TABLE 3

AEROSTRUCTURAL OPTIMIZATION RESULTS

\begin{tabular}{llllll}
\hline \hline & \multicolumn{5}{c}{ Aerostructural Ocptimization } \\
\hline Function/Variable & Initial Value & Optimized Value & Lower bound & Upper Bound & Unit \\
\hline Sink Speed & 0.7 & 0.44 & - & - & $\mathrm{m} / \mathrm{s}$ \\
Glide ratio CL/CD & 38.46 & 49 & - & - & - \\
Aspect ratio & 21.4 & 32.65 & - & - & - \\
Angle of attack & 3 & 7.3 & -10 & 10 & $\mathrm{deg}$ \\
Taper ratio & 1 & 0.3 & 0.3 & 1 & - \\
Twist (root) & 0 & 3.37 & -10 & 15 & $\mathrm{deg}$ \\
Twist (25\% span) & 0 & 4.94 & -10 & 15 & $\mathrm{deg}$ \\
Twist (75\% span) & 0 & 11.64 & -10 & 15 & $\mathrm{deg}$ \\
Twist (0.9 span) & 0 & 6.28 & -10 & 15 & $\mathrm{deg}$ \\
Twist (tip) & 0 & -10 & -10 & 15 & $\mathrm{deg}$ \\
Thickness (root) & 0.0044 & 0.0048 & 0.001 & 0.1 & $\mathrm{~m}$ \\
Thickness (25\% span) & 0.0044 & 0.003 & 0.001 & 0.1 & $\mathrm{~m}$ \\
Thickness (50\% span) & 0.0044 & 0.0016 & 0.001 & 0.1 & $\mathrm{~m}$ \\
Thickness (75\% span) & 0.0044 & 0.0011 & 0.001 & 0.1 & $\mathrm{~m}$ \\
Thickness (tip) & 0.0044 & 0.001 & 0.001 & 0.1 & $\mathrm{~m}$ \\
Wing struct weight & 49 & 23.16 & - & - & $\mathrm{kg}$ \\
\hline \hline
\end{tabular}

\section{CONCLUSION}

The proposed methodology used in this research, is to start from simple wing design, yet poses relatively good design configurations. Then, improving this wing design in order to obtain better performance parameters using OAS tool. The findings of the aerostructural optimization indicated improvements in the performance characteristic of the optimized wing. All the changes that optimizer did on the baseline, supported, in a way, the theories and good practices in wing design for both disciplines; aerodynamic and struc- tural. For example, increasing the aspect ratio, the washout twist distribution and beam thickness distribution. Taken these findings in consideration, will offer interesting design perspectives in advanced design phases. With possibility to conduct high fidelity analysis and optimization, besides, involving more design and flight conditions, like different speeds, altitudes, flight phases (climb, thermalling ... etc.).

\section{ACKNOWLEDGMENT}

The authors would like to thank John Jasa for all the support and availability to help with the OAS Code.

\section{REFERENCES}

[1] F. Thomas and J. Milgram, Fundamentals of Sailplane Design. Maryland, MD: College Park Press College Park, 1999.

[2] S. N. Cubero, S. McLernon, and A. Sharpe, "Over-speeding warning system using wireless communications for road signs and vehicles," Journal of Advances in Technology and Engineering Studies, vol. 2, no. 5, pp. 140-155, 2016. doi: 10.20474 /jater-2.5.2

[3] S. Gudmundsson, General Aviation Aircraft Design: Applied Methods and Procedures. Oxford, UK: ButterworthHeinemann, 2013.

[4] T. Kim, B. Choi, J. Park, S. Hong, and P. Sun-Kyu, "Study of the measurement and stability estimation of concrete roadbed slab at high-speed train in bridge-earthwork transition zone," Journal of Advances in Technology and Engineering Research, vol. 2, no. 2, pp. 41-51, 2016. doi: 10.20474/jater-2.2.3

[5] A. Salas and J. Townsend, "Framework requirements for mdo application development," in 7th AIAA/USAF/NASA/ISSMO Symposium on Multidisciplinary Analysis and Optimization, St. Louis, MO, 1998.

[6] E. J. Cramer, J. E. Dennis, Jr, P. D. Frank, R. M. Lewis, and G. R. Shubin, "Problem formulation for multidisciplinary optimization," SIAM Journal on Optimization, vol. 4, no. 4, pp. 754-776, 1994. doi: 10.1137/0804044

[7] J. Gray, K. Moore, and B. Naylor, "Open mdao: An open source framework for multidisciplinary analysis and optimization," in 13th AIAA/ISSMO Multidisciplinary Analysis Optimization Conference, Fort Worth, TX, 2010. 
[8] J. T. Hwang, D. Y. Lee, J. W. Cutler, and J. R. Martins, “Large-scale multidisciplinary optimization of a small satellite's design and operation," Journal of Spacecraft and Rockets, vol. 51, no. 5, pp. 1648-1663, 2014. doi: 10.2514/1.a32751

[9] J. S. Gray, T. A. Hearn, K. T. Moore, J. Hwang, J. Martins, and A. Ning, "Automatic evaluation of multidisciplinary derivatives using a graph-based problem formulation in openmdao," in 15th AIAA/ISSMO Multidisciplinary Analysis and Optimization Conference, Atlanta, GA, 2014.

[10] J. Hwang and J. Martins, “Allocation-mission-design optimization of next-generation aircraft using a parallel computational framework," in 57th AIAA/ASCE/AHS/ASC Structures, Structural Dynamics, and Materials Conference, San Diego, CA, 2016, p. 1662.

[11] R. D. Falck, J. Chin, S. L. Schnulo, J. M. Burt, and J. S. Gray, 'Trajectory optimization of electric aircraft subject to subsystem thermal constraints," in 18th AIAA/ISSMO Multidisciplinary Analysis and Optimization Conference, Denver, C0, 2017.

[12] J. P. Jasa, J. T. Hwang, and J. R. Martins, “Open-source coupled aerostructural optimization using python," Structural and Multidisciplinary Optimization, vol. 57, no. 4, pp. 1815-1827, 2018. doi: 10.1007/s00158-018-1912-8

[13] N. Bons, X. He, C. A. Mader, and J. Martins, "Multimodality in aerodynamic wing design optimization," in 35th AIAA Applied Aerodynamics Conference, Denver, C0, 2017, p. 3753.

[14] L. W. Cook, J. P. Jarrett, and K. E. Willcox, "Horsetail matching for optimization under probabilistic, interval and mixed uncertainties," in 19th AIAA Non-Deterministic Approaches Conference, Grapevine, TX, 2017, p. 0590.

[15] S. Friedman, S. F. Ghoreishi, and D. L. Allaire, "Quantifying the impact of different model discrepancy formulations in coupled multidisciplinary systems," in 19th AIAA Non-Deterministic Approaches Conference, Grapevine, TX, 2017, p. 1950.

[16] J. J. Bertin and M. L. Smith, Aerodynamics for Engineers. Englewood Cliffs, NJ: Prentice hall Englewood Cliffs, 1989.

[17] J. R. Martins, "A coupled-adjoint method for high-fidelity aero-structural optimization," 2002. [0nline]. Available: https://goo.gl/AQdZ7o

[18] A. B. Lambe and J. R. Martins, "Extensions to the design structure matrix for the description of multidisciplinary design, analysis, and optimization processes," Structural and Multidisciplinary Optimization, vol. 46, no. 2, pp. 273-284, 2012. doi: 10.1023/b:opte.0000048536.47956.62

[19] N. M. Alexandrov and R. M. Lewis, "Analytical and computational aspects of collaborative optimization for multidisciplinary design," AIAA Journal, vol. 40, no. 2, pp. 301-309, 2002.

[20] D. Kraft, A Software Package for Sequential Quadratic Programming. New York, NY: John \& Willy Son's, 1988.

[21] G. Kreisselmeier and R. Steinhauser, "Systematic control design by optimizing a vector performance index,"' in Conference on Computer Aided Design of Control Systems. California, CA: Elsevier, 1980.

[22] A. B. Lambe, G. J. Kennedy, and J. R. Martins, "An evaluation of constraint aggregation strategies for wing box mass minimization," Structural and Multidisciplinary Optimization, vol. 55, no. 1, pp. 257-277, 2017. doi: 10.1007/ s00158-016-1495-1

[23] Federal Aviation Administration, Glider Flying Handbook: FAA-H-8083-13A. Washington, D.C, WA: Federal Aviation Administration, 2013.

[24] M. D. Maughmer, "Design of winglets for high-performance sailplanes," Journal of Aircraft, vol. 40, no. 6, pp. 1099-1106, 2003. doi: $10.2514 / 1.10817$

[25] P. Masak, Design of Winglets for Sailplanes. Hobbs, NM: Soaring Society of America, 1993.

[26] D. Rajnarayan, I. Kroo, and D. Wolpert, "Probability collectives for optimization of computer simulations," in 48th AIAA/ASME/ASCE/AHS/ASC Structures, Structural Dynamics, and Materials Conference, Honolulu, HI, 2007. 